\title{
LAS ESPECIES DE CIGARRITAS (HOMIPTERA, CICADELLIDAE) ASOCIADAS A LAS PLANTAS MEDICINALES Y ORNAMENTALES EN ALLPAHUAYO, IOUITOS-PERÚ
}

\author{
Joel VÁSQUEZ y Pedro W. LOZADA² \\ 1 Programa de Investigación en Biodiversidad Amazónica, Instituto de Investigaciones de la Amazonía Peruana, Apartado \\ 784, Iquitos, Perú. E-mail: jvasquez@iiap.org.pe \\ 2 Museo de Historia Natural de la Universidad Mayor de San Marcos, Av. arenales 1256 apartado 140434 lima 14, Perú. \\ plozada57@hotmail.com
}

\section{RESUMEN}

Los resultados del estudio muestran las especies de "cigarritas" en las plantas medicinales y ornamentales del Centro de Investigaciones Allpahuayo. Un total de 4 especies son reportadas, tres de la sub familia Cicadellinae y una de la sub familia Deltocephalinae. Tres especies están asociadas a las plantas medicinales y una a las plantas ornamentales. Macugonalia moesta ha sido la especie más abundante con $63,5 \%$ asociada a seis especies de plantas hospederas, Hortensia similis, con 15,9\%, con cinco especies hospederas, Copididonus hyalinipennis, con 8,2\%, con dos hospederos y Rhaphirrhinus phosphoreus con 7,1\%, con dos plantas hospederas. Las muestras se encuentran depositadas en las colecciones del Museo de Historia Natural de la Universidad Nacional Mayor de San Marcos, Lima, Perú, reportándose por primera vez la presencia de Rhaphirrhinus phosphoreus en Iquitos.

PALABRAS CLAVES: Cicadellidae, cigarritas, plantas hospederas, Loreto, Perú.

\section{THE SPECIES OF CICADAS (HOMIPTERA, CICADELLIDAE) ASSOCIATED WITH MEDICINAL AND ORNAMENTAL PLANTS IN ALLPAHUAYO}

\section{ABSTRACT}

The results show species of leafhoppers of medicinal and ornamental plants from Allpahuayo Research Center. A total of 4 species are reported, 3 of the subfamily Cicadellinae and one of the subfamily Deltocephalinae. 3 species are associated with medicinal plants and ornamental plants. Macugonalia moesta was the most abundant species with $63.5 \%$ associated with 6 species of host plants, Hortensia similis with $15.9 \%$ with 5 host species, with $8.2 \%$ Copididonus hyalinipennis 2 hosts and Rhaphirrhinus phosphoreus with $7.1 \%$ with 2 host plants. The samples are deposited in the collections of the Natural History Museum of the National University of San Marcos, Lima, Peru. We report for the first time the presence of Rhaphirrhinus phosphoreus in Iquitos.

KEYWORDS: Cicadellidae, leafhoppers, host plants, Loreto, Peru. 


\section{INTRODUCCIÓN}

Las investigaciones sobre Cicadellidae se han orientado principalmente a los insectos plagas, debido a que son vectores de enfermedades en la agricultura y fruticultura (Langlitz, 1964; Rasmussen et al., 2003, Álvarez et al., 2012; Dellapé, 2013). La mayoría de estudios realizados en el Perú sobre Cicadellidae están orientados al registro taxonómico (Lozada 1992b, 1997); así como al de nuevas especies de grupos específicos (Lozada 1992c, 1992d; Lozada \& León, 1996). Solo dos registros de Cicadellidae y sus plantas hospederas fueron realizados en el Perú; el primero en la zona de Jenaro Herrera, Departamento de Loreto, en dos tipos de plantas forrajeras (Lozada, 1992a) y la segunda en la zona de Chanchamayo y Satipo en el Departamento de Junín, sobre frutales de interés comercial y malezas (Lozada \& Arellano, 2008). En este artículo se reporta la presencia de los Cicadellidae asociados a nueve plantas medicinales y dos plantas ornamentales de interés comercial en la Amazonía: Dieffenbachia oblicua "patiquina", Ayapana palustris "caguena", Heliotropium indicum "alacrán", Kalanchoe pinnata "hoja de aire", Aristolochia iquitensis "bufeillo", Ocimum basilicum "albahaca", Wedelia triloba "botón de oro", Hyptis mutabilis "verbena blanca", Lippia dulcis "menta dulce", Teliostachya lanceolata "toe negro", Heliconia caribaea X Heliconia bihai "heliconia roja", y Heliconia psittacorum X Heliconia spathocircinata "heliconia amarilla".

\section{MATERIALES Y MÉTODOS}

El trabajo fue realizado entre los meses de enero y agosto del 2013, en el Jardín de Plantas Medicinales del Centro de Investigaciones Allpahuayo (CIA), ubicado en el $\mathrm{km} \mathrm{26,5}$ de la carretera Iquitos - Nauta, entre las coordenadas S $3^{\circ}$ 58' 038", W 73 25' 136" a 148 msnm., distrito de San Juan, provincia de Maynas, Región Loreto. La temperatura promedio es de $26^{\circ} \mathrm{C}\left(30,7^{\circ} \mathrm{C}\right.$ max., $21,30{ }^{\circ} \mathrm{C} \min$ ) y la precipitación promedio es de $2.950 \mathrm{~mm}$ anual. La colecta ha sido realizada mediante el uso de la red entomológica, aplicando el método del barrido sobre las plantas medicinales, luego del barrido las muestras fueron colocadas en bolsas de polietileno, se rotularon y se depositó en su interior un pedazo de algodón impregnado con alcohol al $70 \%$ para matar los especímenes. Luego se procedió al traslado del material colectado al laboratorio del CIA para la separación de los especímenes, eliminando las hojas, ramitas y otros restos existentes. Posteriormente los Cicadélidos fueron separados de los otros órdenes y se colocaron en alcohol al 70\% para su conservación y posterior caracterización e identificación. Los especímenes colectados fueron caracterizados con la ayuda de un estereoscopio con micrométrico, observando de forma detallada la morfología externa de cada región corporal de los insectos, registrando la forma, color y tamaño. Los ejemplares de todas las especies se encuentran en las colecciones del Museo de Historia Natural de la Universidad Mayor de San Marcos y la determinación taxonómica estuvo a cargo del segundo autor, basada en claves taxonómicas y comparaciones de ejemplares de las colecciones científicas del Museo de Historia Natural de la universidad de San Marcos.

\section{RESULTADOS Y DISCUSIÓN}

Composición de las especies de Cicadellidae. Se determinaron un total de cuatro especies de "cigarritas" en nueve plantas medicinales y una especie en dos plantas ornamentales pertenecientes a dos subfamilias.

Abundancia relativa de las especies dominantes en las plantas evaluadas. En la tabla 1 y 2 se muestra, en porcentajes, la abundancia relativa de los Cicadellidae dominantes asociados a las plantas medicinales y ornamentales. Macugonalia moesta fue la más abundante, alcanzando un porcentaje poblacional de $63,5 \%$ del total de la muestra. La caguena fue la planta con mayor porcentaje de presencia de M. moesta, con 33,9\% y el alacrán fue la planta con menor porcentaje, con 0,6 $\%$. La segunda especie con mayor porcentaje fue Hortensia similis, con $15,9 \%$ y cinco hospederos, alcanzando su mayor porcentaje en "hoja de aire" $7,1 \%$, siendo su presencia baja en "caguena" y "patiquina", con $0,6 \%$. La especie con menor porcentaje fue Copididonus hyalinipennis, con $8,2 \%$ y solo dos hospederos. Con respecto a las plantas ornamentales solo se registró una especie, Rhaphirrhinus phosphoreus, que está asociada a las dos heliconias evaluadas, encontrándose su mayor porcentaje en la heliconia roja, con $7,1 \%$.

\section{Observaciones efectuadas}

\section{Plantas medicinales}

\section{Cicadellinae}

\section{Hortensia similis (Walker, 1851)}

"Cigarrita" de 4,6 $\mathrm{mm}$ de longitud, alas de color verde, el tórax y la cabeza de color amarillo tenue con pequeñas manchas negras a manera de moteado (Figura 1a). Está asociada a: Ayapana palustris "caguena", Dieffenbachia oblicua "patiquina" y Kalanchoe pinnata "hoja de aire, Aristolochia 
iquitensis "bufeillo" y Teliostachya lanceolata "toe negro". Se les ha observado alimentándose de la savia de las hojas tiernas, no se ha observado desecamiento de las hojas. Es una especie muy común en toda la América Tropical. En el Perú se distribuye en Costa, Sierra y Selva, asociada a gramíneas (Lozada, 1997; Lozada \& Arellano, 2008). Por primera vez fue reportada en Axonopus scoparius "maicillo" en Jenaro herrera, para el departamento de Loreto (Lozada, 1992a). Lozada \& Arellano (2008) reportan su presencia en hierba no determinada y en hojas de cafeto y paltos en el fundo Génova en la provincia de Chanchamayo. Costa \& Lozada (2010) registran su presencia en varias localidades de la provincia de la Convención en el departamento del Cusco, no reportan ningún hospedero debido a que fueron colectados con trampas luminosas. Dellapé (2013) reporta a $H$. similis como uno de los vectores potenciales de la bacteria Xylella fastidiosa que causa la enfermedad llamada Clorosis Variegada de los Cítricos (CVC), que afecta a todas las variedades comerciales de naranja dulce y otras especies citrícolas en Argentina y Garita-Cambronero et al., (2008) reporta su presencia en zonas productoras de café afectadas por Xylella fastidiosa en Costa Rica.

\section{Cicadellinae}

\section{Macugonalia moesta (Fabricius, 1803)}

"Cigarrita" de color azul con dos manchas dorsales a manera de banda de color azul en el centro y negro a los costados, la cabeza y el tórax de color negro, en el tórax presenta una banda azul que termina cerca de ojos compuestos y en la cabeza una banda blanca ubicada entre los ojos que cubre los dos ocelos, mide 6,7 mm de longitud (Figura 1b). Fue encontrada en: Wedelia triloba "botón de oro", Ayapana pallustris "caguena", Heliotropium indicum "alacrán", Ocimum basilicum "albahaca", Hyptis mutabilis "verbena blanca" y Aristolochia iquitensis "bufeillo". Estos insectos succionan la savia de las hojas y tallos tiernos, a pesar de que sus poblaciones suelen ser considerables, aún no se ha observado desecamiento severo de los brotes de sus hospederos. Es la especie de Cicadellidae más común en la selva peruana, registrándose en Chanchamayo e Iquitos, sin registro de hospedero
(Lozada, 1997). Lozada \& Arellano (2008) reportan a $M$. moesta en hierbas sin especie determinada; encontrada en un fundo denominado Génova en la provincia de Chanchamayo.

\section{Deltocephalinae \\ Copididonus hyalinipennis (Stál, 1859).}

Esta "cigarrita" presenta el tórax, la cabeza y las patas de color marrón. Los ojos compuestos son de color negro. Presenta dos manchas semicirculares de color negro en la parte dorsal de la cabeza, entre los ojos compuestos. El ala anterior presenta coloración marrón y la mitad posterior transparente mide $5 \mathrm{~mm}$ de longitud, con contextura delgada (Figura 1c). Está asociada a Dieffenbachia oblicua "patiquina" y Heliotropium indicum "alacrán". Se alimentan de la savia de las hojas y tallos, provocando pequeñas manchas circulares. Lozada (1992a) reporta su presencia sobre Axonopus scoparius "maicillo" y Pueraria phaseoloides "kudzu" en Jenaro Herrera, departamento de Loreto y en San Ramón en el departamento de Junín (Lozada, 1997). Linnavuori (1959) reporta la presencia de C. hyalinipennis en Panicum barbinode en Venezuela.

\section{Plantas ornamentales \\ Cicadellinae \\ Rhaphirrhinus phosphoreus (Linneus, 1758)}

"Cigarrita" de color negro con el cuerpo alargado, los ojos de color rojo, la cabeza tiene una estructura a manera de cuerno sobresaliente, las patas son de color amarillo. Mide aproximadamente $12 \mathrm{~mm}$ (Figura 1d). Ha sido encontrada en Heliconia caribaea, X Heliconia bihai "heliconia roja" y Heliconia psittacorum X Heliconia spathocircinata "heliconia amarilla". Se alimenta de la savia de las hojas, sus picaduras ocasionan pequeñas manchas circulares, al inicio las manchas son de color amarillo tornándose posteriormente de color marrón claro con un halo amarillento. Es el primer reporte de la presencia de $R$. phosphoreus para Iquitos (Loreto). Lozada (1997) indica un registro para Perú sin localidad conocida y Costa \& Lozada (2010) reportan su presencia en la localidad de Salvación (Madre de Dios). 
Tabla 1. Especies de Cicadellidae y su distribución en las plantas medicinales del Centro de Investigaciones Allpahuayo, Loreto, Perú. Acumulado en una sesión de colecta para cada planta a comienzos de la época húmeda (enero del 2013) y finales de la época seca (agosto 2013) (+ = presente, - = ausente).

\begin{tabular}{lrrrrrrrrr}
\hline \multicolumn{1}{c}{ Especie } & $\begin{array}{c}\text { Botón de } \\
\text { oro }\end{array}$ & & & $\begin{array}{c}\text { Verbena } \\
\text { blanca }\end{array}$ & Bufeillo & $\begin{array}{c}\text { Hoja del } \\
\text { aire }\end{array}$ & Patiquina Alacrán & $\begin{array}{c}\text { Toe } \\
\text { negro }\end{array}$ \\
\hline Cicadellinae & + & & & & & & & & \\
Macugonalia moesta & $17,6 \%$ & $+33,5 \%$ & $+5,3 \%$ & $+2,4 \%$ & $+4,1 \%$ & - & - & $+0,6 \%$ & - \\
$\begin{array}{l}\text { Hortensia similis } \\
\text { Deltocephalinae }\end{array}$ & - & $0,6 \%$ & - & - & $+4,7 \%$ & $+7,1 \%$ & $+0,6 \%$ & - & $+2,9 \%$ \\
\begin{tabular}{l} 
Copididonus hyalinipennis \\
\hline
\end{tabular} & - & - & - & - & - & - & $+5,3 \%$ & $+2,9 \%$ & - \\
\hline
\end{tabular}

Tabla 2. Especies de Cicadellidae y su distribución en las plantas ornamentales del Centro de Investigaciones Allpahuayo, Loreto, Perú. Acumulado en una sesión de colecta para cada planta a comienzos de la época húmeda (enero del 2013) y finales de la época seca (agosto 2013) $(+=$ presente, $-=$ ausente).

\begin{tabular}{lcc}
\hline \multicolumn{1}{c}{ Especie } & Heliconia roja & Heliconia amarilla \\
\hline Cicadellinae & & \\
Macugonalia moesta & - & - \\
Hortensia similis & - & - \\
Rhaphirrhinus phosphoreus & $+7,1 \%$ & $+5,3 \%$ \\
Deltocephalinae & & - \\
$\quad$ Copididonus hyalinipennis & - & \\
\hline
\end{tabular}



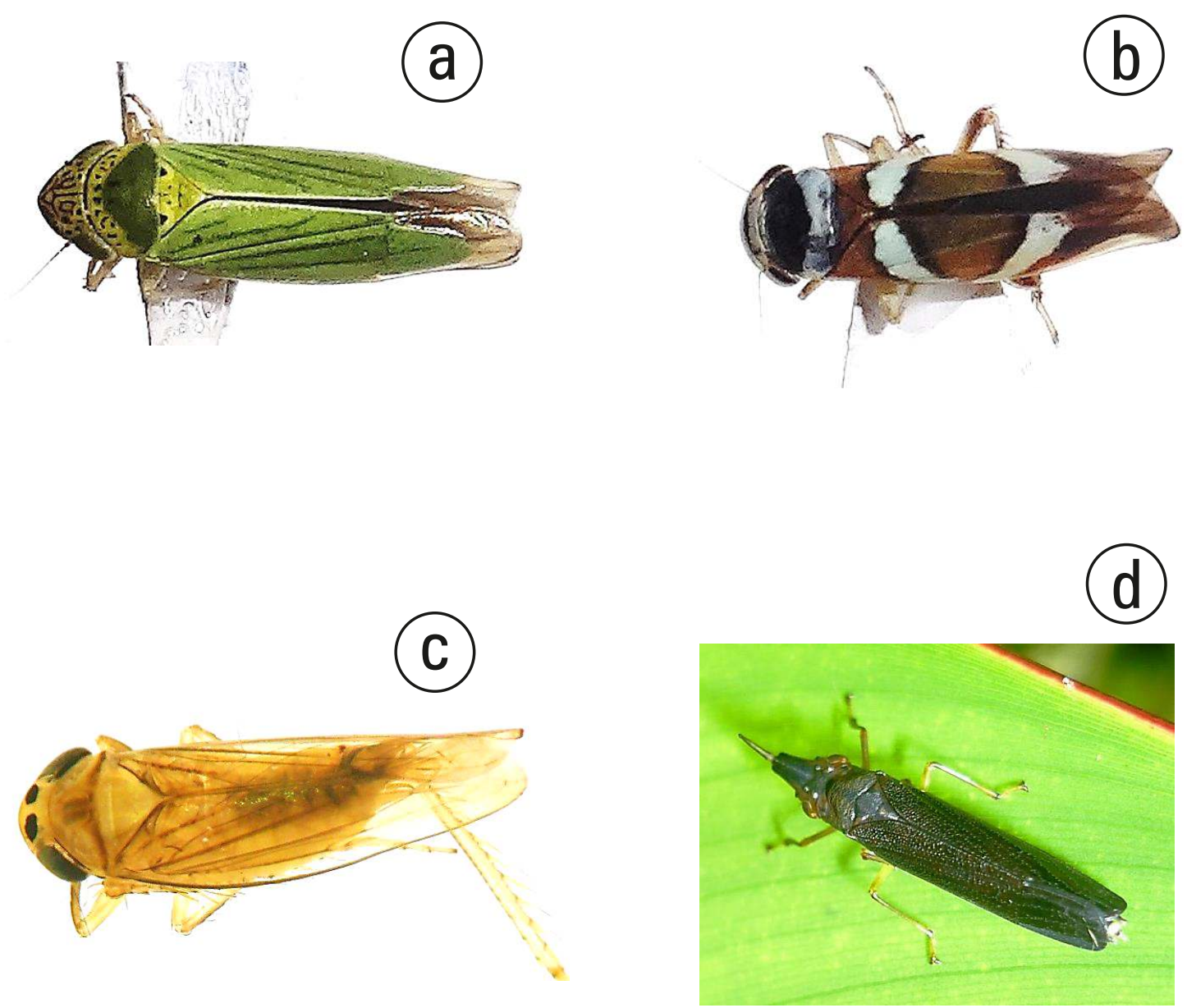

Figura 1. a) Hortensia similis, b) Macugonalia moesta, c) Copididonus hyalinipennis, d) Rhaphirrhinus phosphoreus sobre hoja de Heliconia caribaea X Heliconia bihai "heliconia roja" 


\section{CONCLUSIONES}

Es el tercer reporte de Cicadellidae asociadas a sus plantas hospederas en una zona específica para el Perú. La primera es reportada por Lozada (1997) donde indica las especies de Cicadellidae presentes en "maicillo" y "kudzu", en la zona de Jenaro Herrera, en el Departamento de Loreto; la segunda es reportada por Lozada \& Arellano (2008), indicando las especies de Cicadellidae presentes en café, papaya, palto, cítricos, papaya, piña y malezas (hierbas) de la zona de Chanchamayo y Satipo en el Departamento de Junín. Se reportan once nuevos registros de plantas hospederas para estos Cicadellidae en el Perú, cinco para Hortensia similis, seis para Macugonalia moesta, dos para Copididonus hyalinipennis y dos para Rhaphirrhinus phosphoreus. La especie Rhaphirrhinus phosphoreus se anota como nuevo registro para Loreto. Aunque aún no se han encontrado daños considerables en las plantas hospederas, es preciso mencionar la importancia vectorial de estos insectos señalados como transmisores de microorganismos fitopatógenicos y por el daño mecánico que pueden causar a la vegetación cuando su población es muy alta.

\section{AGRADECIMIENTO}

Este trabajo fue financiado con fondos del Instituto de Investigaciones de la Amazonia Peruana-IIAP, a través del Programa de Investigación en Biodiversidad Amazónica-PIBA. Los autores agradecen a Milca Gianira Elespuru Shuña y Janina Tello Lozano por el apoyo brindado durante el estudio. Al Biólogo Ricardo Zarate Gómez y la Bióloga Elsa Rengifo Salgado por el apoyo en la determinación taxonómica de las plantas hospederas.

\section{REFERENCIAS BIBLIOGRÁFICAS}

Alvarez D. M.; Arroyo W. Y.; Pérez A. M.; Beltrán J. B. 2012. Oviposición y aspectos biológicos del huevo de Oncometopia clarior (Hemiptera: Cicadellidae) en Dioscorea rotunda. Temas Agrarios 17 (2): 77-82.

Costa J. F. \& P. W. Lozada. 2010. Lista de cigarritas (Hemiptera: Cicadellidae) de Cusco, Perú Checklist of leafhoppers (Hemiptera: Cicadellidae) from Cusco. Rev. peru. biol. 17(3): $303-316$.
Dellapé J. 2013. Cicadelinos potenciales vectores de patógenos en cultivos citrícolas del NE argentino. Estudios taxonómicos y moleculares (Insecta: Hemiptera: Cicadellidae). Tesis doctoral. Universidad Nacional de La Plata. Argentina. 227pp.

Garita-Cambronero J.; Villalobos W.; Godoy C.; Rivera C. 2008. Diversidad de Cicadélidos y Clastoptéridos (Hemiptera) en Tres Zonas Productoras de Café Afectadas por Xylella fastidiosa Wells et al. En Costa Rica. Neotropical Entomology 37(4):436-448.

Langlitz E.R. 1964. The Economic Species of Empoasca in the Coastal and Sierra Regions of Perú. Rev. Per. Ent. 7: 54-70.

Lozada P.W. 1992a. Notas sobre Cicadellidae (Homoptera) en plantas forrajeras de Loreto, Perú. Rev. Per. Ent. 35: 24-26.

Lozada P.W. 1992b. Cicadellidae (Homoptera) registrados para el Perú. I: Xestocephalinae, Agalliinae y Deltocephalinae. Rev. Per. Ent. 35: 27-30.

Lozada P.W. 1992c. Una nueva especie de Ladoffa Young, 1977 (Homoptera: Cicadellidae). Rev. Per. Ent. 34: 61-62.

Lozada P.W. 1992d. Una nueva especie de Tortigonalia Young, 1977 (Homoptera: Cicadellidae). Rev. Per. Ent. 34: 63-64.

Lozada P.W.; León C. A. 1996. El Género Cephalogonalia Evans, 1947 (Homoptera: Cicadellidae) en el Perú. Rev. Per. Ent. 39: 11-12.

Lozada P.W. 1997. Cicadellidae (Homoptera) registrados para el Perú. II: Iassinae, Gyponinae y Cicadellinae. Rev. Per. Ent. 40:27-36.

Lozada P.W.; Arellano G. A. 2008. Lista preliminar comentada de las "Cigarritas" (Insecta: Hemiptera: Cicadellidae) en Chanchamayo y Satipo, Ecologia Aplicada, 7(1, 2): 117-122.

Lozada P.W.; Costa J. 2010. Lista de cigarritas (Hemiptera: Cicadellidae) del Cusco, Perú. Rev. Peru. Biol. 17(3): 303-316.

Rasmussen C.; Lagnaoui A.; Esbjerg P. 2003. Advances in the Knowledge of Quinoa Pests. Foods Review International 19 (1,2): 61-75.

Recibido: 26/05/2014

Aceptado para publicación: 30/07/2014 